\title{
PREVALÊNCIA DE FATORES DE RISCO EM CARDIOPATAS DO SETOR DE REABILITAÇÃO CARDÍACA DA FCT/UNESP
}

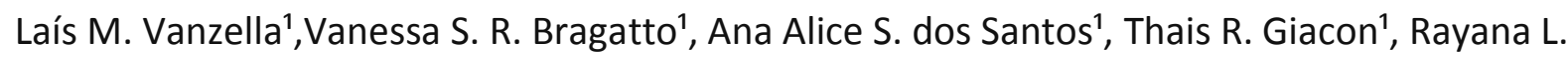
Gomes', Natália Turri da Silva', Camila Balsamo Gardim', Anne Kastelianne Franca da Silva', Naiara Maria de Souza', Luiz Carlos M. Vanderlei'.

${ }^{1}$ Universidade Estadual Paulista Júlio de Mesquita Filho - UNESP - Presidente Prudente - SP. E-mail: lais.vanzella@ig.com.br

\section{RESUMO}

Doenças cardiovasculares (DCV) são as principais causas de morte no Brasil, influenciadas por vários fatores de riscos como a obesidade, hipertensão arterial ( $\mathrm{HA})$, diabetes mellitus, dislipidemia, sedentarismo, dentre outros. Portanto, o trabalho teve como objetivo analisar os fatores de risco presentes em indivíduos participantes do setor de reabilitação cardíaca do Centro de Estudos e Atendimento em Fisioterapia e Reabilitação (CEAFiR). Para isso, foi aplicado um questionário para identificação e anamnese, mensurados pressão arterial, peso e altura para obtenção do IMC, e a taxa de glicose. Diante disto, verificou-se que $56 \%$ dos indivíduos apresentaram histórico familiar de DCV, 81\% apresentam HA, 72,2\% encontraram-se obesos e com sobrepeso, 35,2\% apresentaram glicemia de jejum alterada e 5,8\% encontraram-se com colesterol total e triglicérides alterado. Conclui-se que há alta prevalência de FR no grupo avaliado, ressaltando a grande importância de programas de prevenção para DCV e orientações quanto a mudanças no estilo de vida.

Palavras-chave: hipertensão arterial, obesidade, fatores de risco, doenças cardiovasculares, prevenção e controle

\section{INTRODUÇÃO}

As doenças cardiovasculares (DCV) são uma das principais causas de morte no Brasil, correspondendo a $30 \%$ dos óbitos nas diversas faixas etárias, além de representarem as principais causas de permanência hospitalar prolongada e serem responsáveis pela principal alocação de recursos públicos em hospitalizações ${ }^{1,2}$. São influenciadas por um conjunto de fatores de risco (FR), sendo alguns considerados não modificáveis como a idade e o histórico familiar, e outros modificáveis mediante alterações no estilo de vida como a hipertensão arterial (HA), diabetes mellitus (DM), dislipidemia, obesidade, sedentarismo, estresse, tabagismo e etilismo ${ }^{3}$.

A HA é considerada um problema de saúde pública por sua magnitude, risco e dificuldades no controle. É uma afecção comum, assintomática, prontamente detectável, em geral de fácil tratamento e que costuma gerar complicações letais quando não tratada. Apresenta custos médicos e socioeconômicos elevados, decorrentes principalmente das suas complicações, como: 
doença cerebrovascular, doença arterial coronariana, insuficiência cardíaca, insuficiência renal crônica e doença vascular periférica ${ }^{4}$.

Em relação à obesidade, que no Brasil já atinge mais de $30 \%$ da população adulta ${ }^{5}$, sabe-se que suas causas são de origem multifatorial resultando da interação de fatores genéticos, metabólicos, sociais, comportamentais e culturais. Na maioria dos casos, associa-se ao abuso da ingestão calórica e ao sedentarismo ${ }^{6,7}$.

A DM se caracteriza por hiperglicemia resultante de defeitos na secreção da insulina, ação da insulina ou ambos que acarreta anomalias no metabolismo de carboidratos, gorduras e proteínas $^{8}$. Nas últimas décadas, a DM tem se tornado um sério e crescente problema de saúde pública nos países desenvolvidos e em desenvolvimento, devido ao aumento de sua prevalência, morbidade e mortalidade ${ }^{9}$.

No que diz respeito a dislipidemia, pode-se afirmar que são caracterizadas por distúrbios nos níveis de lipídios circulantes com ou sem repercussão sobre o território vascular, associadas a manifestações clínicas diversas podendo ser influenciadas por distúrbios genéticos e/ou adquiridos $^{10}$.

Diante do aumento das prevalências dos fatores de risco e para conhecer como eles se apresentam em uma população específica bem como para permitir melhor forma de abordagem na orientação, educação e conscientização, torna-se importante efetuar estratégias que permitam o seu controle, como a implementação de programas de prevenção primária e secundária contra os FR para DCV ${ }^{11}$.

Desta forma, o objetivo do presente trabalho foi analisar os FR em indivíduos participantes do setor de reabilitação cardíaca do Centro de Estudos e Atendimento em Fisioterapia e Reabilitação (CEAFiR) da FCT/UNESP.

\section{METODOLOGIA}

Participaram da pesquisa 36 indivíduos, sendo 11 mulheres e 25 homens, pertencentes ao programa de reabilitação no setor de cardiologia do CEAFiR. Os procedimentos deste estudo foram aprovados pelo Comitê de Ética em Pesquisa da FCT/UNESP (CAAE: 17442413.0.0000.5402).

Foram incluídos no estudo pacientes que apresentavam algum tipo de cardiopatia ou FR para DCV e que estavam em tratamento fisioterápico. Já os pacientes que não conseguiram entender e nem responder as perguntas realizadas, assim como não foram capazes de realizar a avaliação completa foram excluídos. 
Inicialmente foi aplicado um questionário com o objetivo de obter informações quanto à identificação e anamnese dos voluntários, onde foram questionados os seguintes itens: idade, presença de DCV, uso de medicamentos, histórico familiar de DCV, presença de FR como HA, DM e dislipidemia. Caso exista a presença, investigar já há quanto tempo o mesmo se apresenta e como é controlado.

A pressão arterial sistólica (PAS) e a pressão arterial diastólica (PAD) (em $\mathrm{mmHg}$ ) foram obtidas de modo indireto com o uso de esfigmomanômetro anaeróide, e classificadas segundo a VI Diretrizes Brasileira de Hipertensão Arterial ${ }^{12}$; foram classificados como hipertensos os indivíduos que apresentavam pressão sistólica $\geq 140 \mathrm{mmHG}$ e pressão diastólica $\geq 90 \mathrm{mmHg}$, ou aqueles que estavam em uso de medicação anti-hipertensiva. A pressão arterial foi aferida apenas uma vez, após aplicação do questionário inicial.

Sequencialmente, foi realizada uma avaliação física composta por obtenção do peso e altura. O peso foi obtido por meio de uma balança digital (Welmy R/I 200 - Brasil), com os indivíduos em posição ortostática com os braços estendidos ao longo do corpo, com roupas leves e sem calçados. Para a obtenção da estatura foi utilizado um estadiômetro (Sanny - Brasil) e para esta medida os voluntários estavam descalços, com os ombros e pescoço alinhados. A partir das medidas de massa corporal e estatura, o índice de massa corporal (IMC) foi calculado utilizando-se a fórmula da massa do indivíduo (quilogramas), dividida por sua altura (metros) ao quadrado, conforme a Diretriz Brasileira de Obesidade ${ }^{13}$.

A taxa de glicose foi verificada pelo exame de punção de polpa digital através do capilar, utilizando-se o aparelho One Touch (Johnson, Argentina). Foram considerados predispostos ao DM os indivíduos que, no exame, apresentaram valores de glicemia de jejum acima de $100 \mathrm{mg} / \mathrm{dl}$, de acordo com a Diretriz da Sociedade Brasileira de Diabetes ${ }^{9}$. Enquanto que, triglicérides e colesterol foram verificados da mesma forma utilizando-se o aparelho Accutrend CGT-BM (Roche, Germany), sendo que foram considerados portadores de colesterol total alterado os indivíduos que apresentaram valores $\geq 160 \mathrm{mg} / \mathrm{dl}$, e portadores triglicérides os indivíduos que apresentaram valores $\geq 150 \mathrm{mg} / \mathrm{dl}$, de acordo a IV Diretriz Brasileira sobre Dislipidemias e Prevenção da Aterosclerose ${ }^{14}$.

Os dados obtidos foram organizados em planilha computacional e analisados por meio de estatística descritiva. 


\section{RESULTADOS}

Foram analisados dados de 36 indivíduos com média de idade: $66,1 \pm 9,3$ anos. Na tabela 1 estão apresentadas as características físicas da amostra.

Tabela 1. Caracterização amostral composta por idade, altura, peso e índice de massa corporal.

\begin{tabular}{cc}
\hline & Indivíduos (N 36) \\
\hline Idade & $66,1 \pm 9,3$ \\
Altura & $1,66 \pm 1,0$ \\
Peso & $79,1 \pm 17,9$ \\
IMC & $28,6 \pm 6,4$ \\
\hline
\end{tabular}

Média \pm desvio padrão, $\mathrm{N}$ = numero absoluto, IMC = índice de massa corporal

No tabela 2, encontra-se apresentado a prevalência de FR nos pacientes do setor de reabilitação cardíaca do CEAFiR.

Tabela 2. Prevalência de fatores de risco de indivíduos frequentadores do setor de reabilitação cardíaca do CEAFiR

\begin{tabular}{ccc}
\hline & N & (\%) \\
\hline Histórico familiar & 20 & 56 \\
Hipertensão Arterial & 29 & 81 \\
\hline Obesidade e Sobrepeso & 26 & 72,2 \\
Glicemia de jejum alterada & 11 & 35,2 \\
Colesterol total alterado & 2 & 5,8 \\
\hline Triglicérides alterado & 2 & 5,8
\end{tabular}

$\mathrm{N}=$ número absoluto. Hipertensão arterial: considerando valores acima de 140/90mmHg pela VI Diretrizes brasileiras de hipertensão arterial. Sobrepeso e obesidade: considerando valores de índice de massa corporal acima de $25 \mathrm{~kg} / \mathrm{m}^{2}$ pela Diretrizes Brasileiras de Obesidade. Nível de glicemia alterado: considerando valores de glicemia $>100 \mathrm{ml} / \mathrm{dl}$ pela Diretriz brasileira de Diabetes. Colesterol alterado: considerados valores a partir de $160 \mathrm{mg} / \mathrm{dl}$ e triglicérides alterado: valores a partir de $150 \mathrm{mg} / \mathrm{dl}$

\section{DISCUSSÃO}

No presente estudo verificou-se a alta prevalência de FR dos indivíduos que frequentam um setor de reabilitação cardíaca, sendo que 56\% apresentaram histórico familiar de DCV, $81 \%$ eram hipertensos mesmo que controlados por medicamento, $72,2 \%$ foram considerados obesos ou com sobrepeso, 35,2\% apresentavam glicemia de jejum alterada e 5,8\% encontraram-se com o colesterol total e triglicérides alterados. 
Em um estudo realizado por Girotto et al em uma Unidade de Saúde da cidade de Londrina com indivíduos de 20 a 79 anos, encontrou-se que 30,9\% dos indivíduos apresentavam histórico familiar para $\mathrm{DCV}^{15}$. Em nosso estudo, foi encontrado que $56 \%$ dos pacientes apresentaram este FR, desta forma tem-se que nossos valores foram mais elevados, e isto pode ser devido a nossa população já ser cardiopata.

Em relação à hipertensão arterial, que é uma das patologias de maior prevalência na população adulta e principalmente nos idosos ${ }^{16}$, encontramos que $81 \%$ dos indivíduos são considerados hipertensos. Em um estudo epidemiológico realizado por Miranda et al com idosos residentes na cidade de São Paulo foi encontrado prevalência de hipertensão arterial de 62\% dos indivíduos, relatando que esse número pode ser devido às alterações próprias do envelhecimento que reduzem a elasticidade arterial, induzindo ao aumento da resistência periférica e tornando o indivíduo mais propenso ao desenvolvimento dessa doença crônica ${ }^{17}$.

Já se tratando da obesidade sabe-se que esta vem se tornando uma epidemia global. De acordo com os dados recém publicados nos Estados Unidos, 67\% da população adulta sofre de sobrepeso, enquanto 34\% apresentam obesidade representando um aumento de $75 \%$ em relação a $1991^{18}$. Em nosso estudo a prevalência encontrada foi de $72,2 \%$ quando somados sobrepeso e obesidade, o que evidencia mais uma vez a grande incidência deste fator de risco, condizente também com outros autores.

Um resultado preocupante foi a prevalência encontrada para o nível de glicose alterado em $35,2 \%$ dos indivíduos avaliados, prevalência um pouco acima da encontrada na literatura. Gus et al. ${ }^{19}$ encontrou prevalência de $7 \%$ para glicemia alterada em adultos acima de 20 anos mas considerou valores acima de $126 \mathrm{mg} / \mathrm{dl}$, Pansani et $\mathrm{al}^{20}$ encontrou prevalência de $11,54 \%$ para os mesmos valores de Gus em mulheres acima de 60 anos.

No que se refere a dislipidemia, um estudo realizado com os usuários do Sistema Único de Saúde de Goiânia apresentou que $23,4 \%$ da população estudada apresenta-se dislipidêmica, visto que foi levado em consideração as pessoas que utilizavam medicamentos para a patologia e aquelas que apresentavam diagnóstico médico ${ }^{21}$. Em nosso estudo apenas 5,8\% dos indivíduos encontraram-se com alterações nos níveis de colesterol total e triglicérides quando houve realização do exame de punção da polpa digital, mas tem-se que boa parte destes indivíduos utilizam medicamentos para controlar a patologia.

Portanto o presente estudo mostrou a alta prevalência de FR em indivíduos que participam de um programa de reabilitação cardíaca. Diante disso, é necessária a intensificação de programas 
de controle de HA, obesidade e sobrepeso, DM e dislipidemia, visando uma diminuição do aparecimento de DCV e melhor qualidade de vida à população.

Outro fator importante que foi evidenciado neste estudo foi o fato de que indivíduos já acometidos por patologias do sistema cardiovascular e que realizam tratamento ambulatorial não estão totalmente privados de complicações neste sistema uma vez que há outros fatores envolvidos na determinação da saúde cardíaca. Por isso programas de prevenção secundária, assim como os de prevenção primária são indispensáveis a fim de promover modificações positivas no estilo de vida e evitar complicações futuras.

\section{CONCLUSÃO}

Podemos observar com base nos resultados acima descritos, que os indivíduos portadores de DCV apresentam um ou mais FR analisados nesta pesquisa. Com isso, ressalta-se a importância de programas de prevenção primária e secundária de DCV e da presença de orientações a respeito das mudanças no estilo de vida.

\section{REFERÊNCIAS}

1. Coltro RS, Mizutani BM, Mutti A, Délia MPB, Martinelli LMB, et al. Frequência de fatores de risco cardiovasculares em voluntários participantes de evento de educação em saúde. Ver Assoc Med Bras. 2009;55(5): 606-10. http://dx.doi.org/10.1590/S0104-42302009000500028

2. Castro LCV, Franceschini SCC, Priore SE, Peluzio MCG. Nutrição e doenças cardiovasculares: os marcadores de risco em adultos. Rev Nutr. 2004; 17(3): 369-77. http://dx.doi.org/10.1590/S1415$\underline{52732004000300010}$

3. Rique $A B R$, Soares $E A$, Meirelles $C M$. Nutrição e exercício na prevenção e controle das doenças cardiovasculares. Ver Bras Med Esporte. V.8, n. 6 - Nov/Dez,2002.

4. Nascente FMN, Jardim PCBV, Peixoto MRGP, Monego ET, Barboso WKS, Moreira HG, et al. Hipertensão arterial e sua associação com índices antropométricos em adultos de uma cidade de pequeno porte do interior do Brasil. Rev Assoc Med Bras. 2009;55(6):716-722. http://dx.doi.org/10.1590/S0104-42302009000600017

5. Mendes MJFL, Alves JGB, Alves AV, Siqueira PP, Freire EFC. Associação de fatores de risco para doenças cardiovasculares em adolescentes e seus pais. Ver Bras Saúde Martern Infant. 2006; 6(1): 549-554.

6. Carneiro G, Faria AN, Ribeiro Filho FF, Guimarães A, Lerário D, et al. Influência da distribuição da gordura corporal sobre a prevalência da hipertensão arterial e outros fatores de risco cardiovascular em indivíduos obesos. Rev Assoc Med Bras. 2003; 49(3); 306-11. http://dx.doi.org/10.1590/S0104-42302003000300036 
7. Tavares TB, Nunes SM, Santos MO. Obesidade e qualidade de vida : Revisão da literatura. Ver Med Minas Gerais 2010; 20(3): 359-366.

8. Organization WH. Definition, Diagnosis and Classification of Diabetes Mellitus and its Complications. Geneva: Department of Noncommunicable Disease Surveillance. 1999.

9. Diretrizes da Sociedade Brasileira de Diabetes 2009/Sociedade brasileira de diabetes.-Itapevi, SP: A. Araújo Silva Farmacêutica, 2009.

10. Coelho VG, Caetano LF, Liberatore Júnior RdelR, Cordeiro JÁ, Souza DRI. Perfil Lipídico e Fatores de Risco para Doenças Cardiovasculares em Estudantes de Medicina. Arquivos Brasileiros de Cardiologia, 2005; 85(1); 57-62. http://dx.doi.org/10.1590/S0066-782X2005001400011

11. Triches RM, Giugliani ERJ. Obesidade, práticas alimentares e conhecimentos de nutrição em escolares. Rev Saúde Pública. 2005;39(4):5417. http://dx.doi.org/10.1590/S0034$\underline{89102005000400004}$

12. VI Diretriz Brasileira de Hipertensão. Rev Bras Hipertens. 2010;17(1):5-6.

13. Diretrizes brasileiras de obesidade 2009/2010 / ABESO - Associação Brasileira para o Estudo da Obesidade e da Síndrome Metabólica. - 3.ed. - Itapevi, SP : AC Farmacêutica, 2009.

14. IV Diretriz Brasileira Sobre Dislipidemias e Prevenção da Aterosclerose. Arq Bras Cardiol. 2007; 88(1):1-19. http://dx.doi.org/10.1590/S0066-782X2007000100001

15. Girotto E, Andrade SM, Cabrera MAS, Ridão EG. Prevalência de fatores de risco para doenças cardiovasculares em hipertensos cadastrados em unidade de saúde da família. Acta Scientiarum 2009; v.31, n.1; 77-82.

16. Barroso WKS, Jardim PCBV, Vitorino PV, Bittencourt A, Miquetichuc F. Influencia da atividade física programada na pressão arterial de idosos hipertensos sobre tratamento não farmacológico. Ver Assoc Med Bras 2008; 54(4): 328-33.

17. Miranda RD, Perrotti TC, Bellinazzi VR, Nóbrega TM, Cendoroglo MS, et al. Hipertensão arterial do idoso: peculiaridades na fisiopatologia, no diagnostico e no tratamento. Ver Bras Hipertens 2002; 9: 293-300.

18. Gomes F, Telo DF, Souza HP, Nicolau JC, Halpern A, et al. Obesidade e Doença Arterial Coronariana: Papel da Inflamação Vascular. Arq Bras Cardiol 2010; 94(2) : 273-279. http://dx.doi.org/10.1590/S0066-782X2010000200021

19. Gus I, Fischmann A, Medina C. Prevalência dos Fatores de Risco da Doença Arterial Coronariana no Estado do Rio Grande do Sul. Arq Bras Cardiol, 2002; v.78, n.5: 478-83. http://dx.doi.org/10.1590/S0066-782X2002000500005 
20. Pansani AP, Anequini IP, Vanderlei LCM, Tarumoto MH. Prevalencia de fatores de risco para doenças coronarianas em idosas frequentadores de um programa "Universidade Aberta à Terceira Idade". Arq Ciênc Saúde 2005; 12(1) : 27-31.

21. Ferreira CCC, Peixoto MRG, Barbosa MA, Silveira EA. Prevalência de Fatores de Risco Cardiovascular em Idosos do Sistema Único de Saúde de Goiânia. Arq Bras Cardiol 2010; 95(5): 621-628. http://dx.doi.org/10.1590/S0066-782X2010005000141 